\title{
Introducing Parallel Zone Picking to Warehouse Batch Picking Systems
}

\author{
Kenji Tanaka ${ }^{1}$, Asaki Ihara ${ }^{1}$ and Jing Zhang ${ }^{2, *}$ \\ ${ }^{1}$ Department of Systems Innovation, The University of Tokyo, Tokyo, Japan \\ ${ }^{2}$ School of Mathematics, Physics and Information Science, Zhejiang Ocean University, China \\ *Corresponding author
}

Keywords: Order batching, Asynchronous, Parallel processing, Batch method, Simulation.

\begin{abstract}
Picking is labor intensive and the most expensive aspect of distribution warehouse operations. Therefore, research on improving the efficiency of distribution warehouse picking has become important. We propose an asynchronous parallel processing system for distribution warehouses with a multiple-zone layout and verify the efficiency improvement by real data.
\end{abstract}

\section{Introduction}

\section{Background}

Picking is labor intensive and the most expensive aspect of distribution warehouse operations. Previous studies for improving the efficiency of various picking methods have been conducted [1]. In particular, many studies on efficiency improvement of the total picking method of putting together orders for multiple shipping destinations have considered this as an Order Batching Problem (OBP). In this method, a zone picking method is assigned to each divided zone.

Total picking is a method in which a picker picks multiple customer orders at once. It is necessary to sort items for each customer order, generally in a pick-and-sort or sort-while-picking procedure [2]. On the other hand, methods in which only one customer order is picked at a time are called single picking methods.

In this research, completing customer orders in total picking is called "batching," and batched customer orders are called "batch orders." In sort-while-picking methods, the picker sorts items for each customer while picking. In this case, there is a sorting area separate from the picking area. Recently, with increased demand for online shopping, picking work volumes can exceed processing capacities assumed at the time of warehouse design. Large online shopping distribution warehouses in particular assume conventional total picking. In methods where one picker covers the whole area, movement distances become inefficient. A combination of total picking and zone picking would be more efficient, but this approach is limited in existing methods. The proposed method addresses this limitation.

This research considers a warehouse consisting of picking, temporary storage, and sorting areas. We define "asynchronous pick-up total picking" as a method for batch order picking that is not affected by other zones. We verify that the proposed method improves processing capacities in distribution warehouses. We also propose an algorithm for improving comprehensive processing capacity, including placement and sorting.

\section{Research Purpose}

Order batch processing efficiency in total picking has been solved as an OBP. OBPs are used to group customer orders based on the arrangement of items in a warehouse and a given picking equipment capacity (batching). In OBPs, the solution is searched for using the movement distance or time necessary to collect all items as an evaluation function. The solution is a set of batch orders, to which all customer orders are assigned. As a constraint, many studies set an upper limit on the amount of items that one picker can carry at one time.

Solutions in OBPs are divided into constructive solutions and meta-heuristics solutions. The former are algorithms that do not reassign customer orders once assigned to a batch order, first proposed by Eslayed et al. [3] in the 1980s. The latter are algorithms that search for improved 
solutions by incorporating a re-assignment step for customer orders. Metaheuristics have been applied to OBP since around 2005 [4]. In these existing studies, objective functions and constraints were simplified. Therefore, practical use is difficult.

Based on present conditions and previous research, this research verifies processing capacity improvements resulting from introducing asynchronous parallel processing in a multi-zone layout in the total picking of a distribution warehouse.

To that end, we perform the following:

- $\quad$ Define an OBP in an asynchronous parallel processing-type total picking method.

Because efficiency problems related to the picking method considered in this research have not been studied, we define this as a new batching problem

- $\quad$ Design and construction of a picking list output system.

We build a system that outputs an efficient solution to the defined problem. As its internal algorithm, we construct algorithms for batch order creation, picking list creation, and picking list ordering. A picking list is a list that indicates the items to be picked by the picker.

\section{Modeling}

There are two primary differences from problem definitions in existing OBPs.

- It is necessary to batch customer orders in two ways, with batch order processing by sorting devices and with picking lists for performing picking together.

- It is necessary to consider the order of outputting picking lists to improve the operation rate of sorting devices.

We uniquely set an evaluation function that evaluates the processing capacity of the asynchronous parallel processing-type total picking method that is the subject of this research. This function is defined as the time required from the start of picking the first order to the end of sorting all orders under a certain input resource, as shown in Equation (1). TI is a function that evaluates processing power in the target system. $T_{\text {work }}$ is the time taken from the start of picking the first order to the end of sorting all orders. $C_{\text {resource }}$ is a cost conversion for the amount of equipment and personnel introduced.

$$
T I=T_{\text {work }} / C_{\text {resource }}
$$

We consider three constraints:

- $\quad$ As in existing OBPs, there is an upper limit on the number of items that the picker can pick in a single picking.

- $\quad$ Unlike existing OBPs, there is an upper limit on storage volume in the temporary storage area.

- Unlike existing OBPs, there is an upper limit on the number of customer orders that the sorting device can sort at one time.

The picking area is divided into multiple zones, and items are placed in each zone. A group of multiple products is called a category, and each zone comprises multiple categories. Picking bases are located in one corner of each zone. Each location has a starting point for a conveyor that leads to the temporary storage location. At the warehouse site, when the picker receives a picking list, it transports it to the conveyor.

\section{Proposal of Picking list Output System}

\section{System Overview}

Figure 1 shows the system flow from receiving a customer order to evaluating processing capacity. The proposed system reads customer orders according to this flow, and outputs picking lists in order. With this system, it is possible to quantitatively evaluate the processing capacity of the targeted picking method. 
First, read customer order data ordered at regular intervals. When the read number becomes a data termination flag, the picking list is output as an input value. Next, create a batch order based on the read customer order. This determines the order in which the sorting device sorts. After that, divide the batch order into zones. Finally, create a picking list based on the divided batch order. The picking list is output by further batching the divided batch orders. This process is based on the premise that the volume of batch orders assigned to each zone is less than the volume that can be performed in one picking.

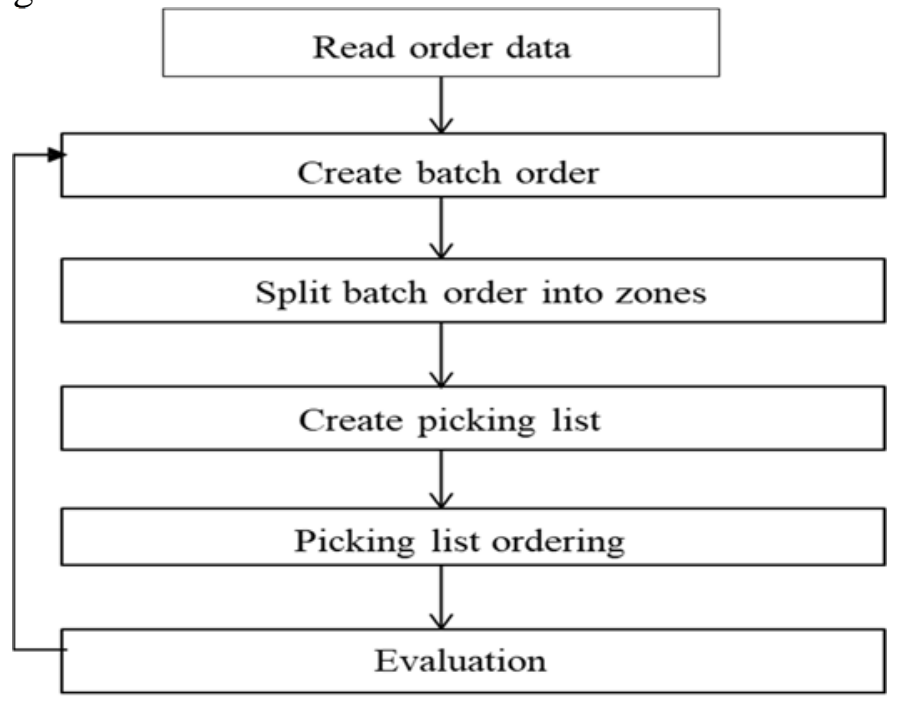

Figure 1. Asynchronous parallel processing-type total picking method flow chart.

The internal algorithm of this system organizes the processing capacity improvement. To improve the processing capacity in the range defined by the evaluation function, it is necessary to make the sorting and picking operations more efficient. We prepared four approaches toward such efficiency improvements:

- $\quad$ Organize customer orders up to the upper limit of sorter capacity.

- $\quad$ Reduce downtime of the sorting device.

- $\quad$ Reduce movement distances for each picking.

- $\quad$ Reduce the number of pickings.

\section{Batch Order Creation Algorithm}

The goal for batch order creation is twofold: preparing customer orders up to the upper limit of sorter capacity, and reducing the travel distance for one picking. To reduce the travel distance for one picking in category-based placement, we apply SeedAlgorithm.

SeedAlgorithm continuously creates batches in two phases. The first phase assigns the first customer order to be newly batched. The second phase keeps assigning as many unassigned customer orders as possible. This creates one batch order. SeedAlgorithm batches all customer orders by continuing to make assignments to unassigned orders. Allocation is based on seed selection rules that determine the first order and on order combination rules that determine additional orders.

The efficiency of a SeedAlgorithm is expressed as the total time required for picking. The time taken for picking can be divided into time spent inside and outside of a category. It is assumed that the required time in the category is proportional to the number of product types, and that the required time outside the category is determined by the round-trip time between the base and the category farthest from the base.

A joint rule is set by giving priority to the category with the fewest additional categories in the batch, and by selecting the category with the shortest movement between additional bases and categories. The following rules for $\mathrm{X}$ and $\mathrm{Y}$ can be considered as policies for selection rules. In the case study, four rules combining X and $\mathrm{Y}$ (X1Y1, X1Y2, X2Y1, X2Y2) are considered. 
- $\quad$ Prioritize clusters with many categories (X1), or prioritize clusters with few categories (X2)

- $\quad$ Prioritize clusters where the farthest category is closest to the base (Y1), or prioritize distant categories (Y2)

\section{Picking List Creation Algorithm}

Divide the created batch order into each zone and create a picking list based on them. The goal is to reduce the number of pickings. In this algorithm, we combine as many batch orders as possible, divided into zones such that this constraint is satisfied.

\section{Picking List Ordering Algorithm}

By using an algorithm in the order of outputting the created picking list, we aim to shorten the nonoperating time of the sorting device.

The condition for operating the sorting device is a completed batch order existing in the temporary storage area. That is, in the case where there is a non-operating sorting device, a divided batch order from some zones has arrived at a temporary storage location but an order from other zones has not arrived, or immediately after the start of picking work. We first avoid picking list output timings for divided batch orders that are extremely separated between zones. Then, we aim to complete batch orders as quickly as possible after the start of picking operations, and propose a picking list ordering algorithm.

\section{Picking Simulation}

We create a time-series picking simulation every minute, allowing us to evaluate the warehouse processing capacity from the viewpoint of both picking and sorting.

\section{System Comparison Evaluation}

We verified and compared the proposed system using data from an Internet mail order company that uses the total picking method, and have established picking, temporary storage, and sorting areas in a warehouse. Simulation evaluations were performed under the following conditions:

- $\quad$ A picker can handle up to 6 units in one picking.

- $\quad$ The sorting device can sort up to 20 customer order items at once.

- $\quad$ The temporary storage area capacity is assumed to be sufficiently large.

- $\quad$ There are 5 sorting devices and 20 pickers.

- $\quad$ The farthest category requires one minute for round-trip travel from the base.

Efficient picking list output was performed for 1,228 customer orders accumulated over an average night-time, and we verified that the processing capacity improved as compared with the current total picking method.

The simulation results in Figure 2 show the superiority of the proposed system.

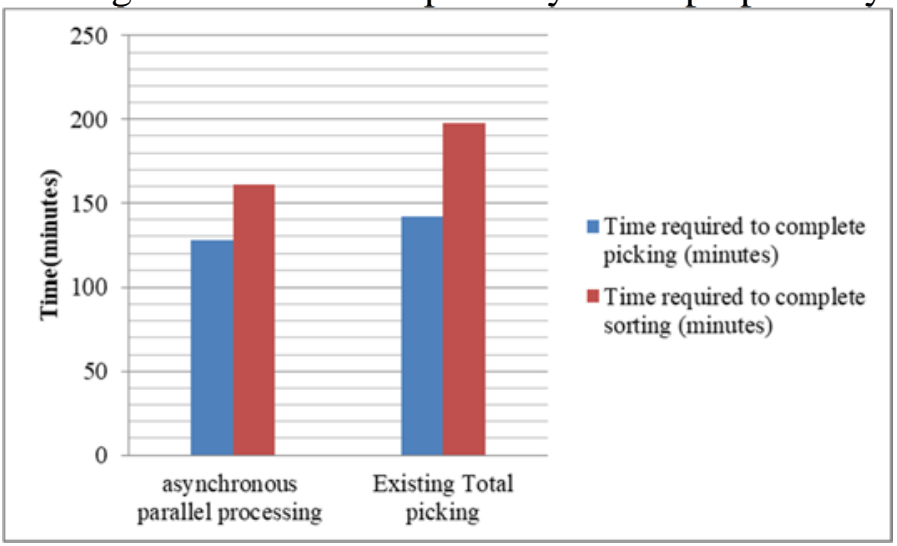

Figure 2. Simulation results of the proposed system. 


\section{Conclusion}

Using an asynchronous parallel processing method for multiple zones, we proposed a reduced order batch method that improves final processing time over existing total picking methods and improves processing capacity in distribution warehouses. We demonstrated the proposed method's effectiveness using actual data.

\section{References}

[1] De Koster, R., Le-Duc, T., \& Roodbergen, K. J. (2007). Design and control of warehouse order picking: A literature review. European Journal of Operational Research, 182(2), 481-501.

[2] Parikh, P. J., \& Meller, R. D. (2008). Selecting between batch and zone order picking strategies in a distribution center. Transportation Research Part E: Logistics and Transportation Review, 44(5), 696-719.

[3] Elsayed, E. A., \& Unal, O. I. (1989). Order batching algorithms and travel-time estimation for automated storage/retrieval systems. The International Journal of Production Research, 27(7), 1097-1114.

[4] Hsu, C. M., Chen, K. Y., \& Chen, M. C. (2005). Batching orders in warehouses by minimizing travel distance with genetic algorithms. Computers in Industry, 56(2), 169-178. 